\title{
Cuantificación del factor de crecimiento similar a la insulina (IGF-I) total en tejidos de rata
}

\author{
Elizabeth Rico, Myriam Sánchez de Gómez
}

\begin{abstract}
Resumen
Se establecieron los contenidos totales de IGF-I en algunos tejidos de rata adulta normal (hígado, riñón, corazón, pulmón, timo, bazo y músculo esquelético) por medio de radioinmunoanálisis $(\mathrm{RIA})$ específico. La efectividad de la extracción ácido-etanólica $(\mathrm{AE})$, corrientemente empleada para eliminar la interferencia de las proteínas de unión del IGF-I (IGFBPS) en el análisis del factor en suero, se evaluó para las muestras de tejidos y se comparó con la extracción $\mathrm{AE}$ seguida de crioprecipitación (AEC). Los resultados mostraron que la extracción $A E$ no permite remover completamente las IGFBPs y que con el paso adicional de crioprecipitación se libera aproximadamente $20 \%$ más de IGF-I de los tejidos. Finalmente, por medio de RIA se estableció la distribución tisular de este factor de crecimiento en rata. El método aquí descrito puede aplicarse en estudios de regulación metabólica o nutricional del IGF-I.
\end{abstract}

\section{Quantification of total insulin-like growth factor (IGF-I) in rat tissues}

Total IGF-I in some tissues (liver, kidney, heart, lung, thymus, spleen and skeletal muscle) from normal adult rats was measured by specific radioimmunoassay (RIA). Acid-ethanol extraction (AE), currently utilised to separate IGF-I binding proteins (IGFBPs) in serum prior to RIA, was assessed in tissue analysis and was compared with $A E$ followed by crioprecipitation. It was found that $A E$ extraction does not completely remove IGFBPs, whereas the additional crioprecipitation step releases approximately $20 \%$ more IGF-I from the tissues. Rat IGF-I tissue distribution was finally established by RIA. The method here described is useful for the study of the metabolic or nutritional IGF-I regulation.

El IGF-I es un péptido de 70 aminoácidos con similitud estructural con la insulina (1). Circula enlazado a proteínas de alta afinidad (IGFBPs), de las cuales 6 han sido clonadas y secuenciadas en rata (2). Junto con sus receptores de tipo I y II y proteasas específicas para IGFBPs constituyen el eje de IGF-I. Este eje está directamente involucrado con las acciones promotoras del crecimiento de la hormona de crecimiento $(\mathrm{GH})(3)$.

El IGF-I es el principal mediador del crecimiento del tejido esquelético, principalmente, aunque posee además una gran variedad de funciones a nivel celular, entre las que se cuentan la progresión del ciclo celular, la proliferación y la diferenciación celular. Todas estas funciones implican el estímulo de la síntesis de ADN, ARN, proteínas y colágeno, entre otros (4-6). El hígado es considerado la mayor fuente de IGF-I en el organismo, aunque el mensajero para este péptido ha sido detectado en gran cantidad de tejidos como el pulmón, el riñón, el corazón y otros $(7,8)$. Esta producción local de IGF-I es muy importante fisiológicamente ya que se puede dar la forma de acción auto/paracrina en contraste a la forma de acción endocrina común a todas las hormonas (5).

Laboratorio de Hormonas, Departamento de Química, Universidad Nacional de Colombia, Santa Fe de Bogotá, Colombia. Recibido para su publicación: 22 de abril de 1998. - Aprobado para su publicación:19 de mayo de 1998. 
La regulación de su producción es dependiente de la GH y del estado nutricional $(4,9)$ y sus niveles son un indicador de la funcionalidad del eje IGF-I/GH.

El método preferido para la cuantificación del IGF-I sigue siendo el radioinmunoanálisis (RIA), aunque una de las mayores dificultades es la interferencia de las proteínas de unión de IGF.

Diversos sistemas se han empleado para la eliminación de dichas proteínas fundamentalmente en suero o plasma, basadas en la disociación de los complejos por tratamiento a $\mathrm{pH}$ ácido y separación de las mismas por precipitación o cromatografía de filtración en gel (10-12). En este trabajo se describen las condiciones para la cuantificación del IGF-I total en tejidos por medio del RIA, previa eliminación de las proteínas de unión (IGFBP). El radioinmunoanálisis, validado estadísticamente en un trabajo previo, se empleó en la cuantificación del IGF-I total en tejidos y sueros de rata normal en crecimiento.

\section{Materiales y métodos}

\section{Animales}

Se utilizaron ratas Winstar macho $(n=5)$ de ocho semanas de edad del bioterio del Departamento de Farmacia de la Universidad Nacional. Los animales se mantuvieron en condiciones de temperatura y humedad controladas y con libre acceso a una dieta estándar y agua.

\section{Estándares hormonales}

IGF-I, DES (1-3)IGF-I receptor grade de Laboratorios GROPEP (Peptides for Growth Ltd., Australia).

\section{Anticuerpos}

Anti-IGF-I y anti-IgG (policlonales) obtenidos en el Laboratorio de Hormonas del Departamento de Química de la Universidad Nacional de Colombia, por inoculación en conejos y cabros, respectivamente. La reactividad cruzada para el anti-IGF-I fue $0,5 \%$ para IGF-II y $0,025 \%$ para insulina y con una constante de afinidad de $0,106 \times 10^{10} \mathrm{l} / \mathrm{mol}$.

\section{Radioisótopo}

$\mathrm{Na}^{125}(17 \mathrm{mCi} / \mu \mathrm{g})$, Amersham, Inglaterra.

\section{Obtención de tejidos y suero de rata}

Los animales se sacrificaron por asfixia con $\mathrm{CO}_{2}$. La sangre se tomó de la vena cava descendente y se obtuvo el suero, el cual se almacenó a $-70^{\circ} \mathrm{C}$. Los órganos se extrajeron e, inmediatamente, se guardaron a $-30^{\circ} \mathrm{C}$ en frascos plásticos, hasta la extracción de IGF-I.

\section{Extracción de IGF-I tisular}

El tejido se pesó, cortó y transfirió a tubos siliconizados. Se adicionaron $5 \mathrm{~mL} / \mathrm{g}$ tejido húmedo de una solución de ácido acético $(0,1 \mathrm{M})$ conteniendo inhibidor de serín proteasas (aprotinina) (13). El tejido se sonicó hasta la disrupción total del mismo. Incubaciones en hielo y centrifugaciones sucesivas $(3.000 \mathrm{rpm}, 30$ min, $4^{\circ} \mathrm{C}$ ), permitieron obtener los sobrenadantes libres de residuos de tejido, los cuales se congelaron $\left(-70^{\circ} \mathrm{C}\right) \mathrm{y}$, posteriormente, se liofilizaron.

\section{Cuantificación}

Se llevó a cabo por radioinmunoanálisis (RIA) específico, siguiendo la técnica estandarizada previamente en el laboratorio (14). La yodinación se llevó a cabo por el método modificado de la cloramina-T, obteniendo porcentajes de incorporación de $60-65 \%$ con actividades específicas de $72-94 \mu \mathrm{Ci} / \mu \mathrm{g}$ IGF-I.

Para la eliminación de las proteínas de unión, se emplearon las técnicas denominadas $\mathrm{AE}$ (ácido-etanol) y AEC (ácido-etanol-crioprecipitación). Para ello, el suero y el extracto de tejido reconstituido en buffer de análisis fueron tratados con una solución ácido-etanol en relación $87,5(\mathrm{HCl} 2 \mathrm{~N}): 12,5$ (etanol absoluto) y las proteínas fueron removidas por centrifugación a 3.000 rpm, 30 minutos, $4^{\circ} \mathrm{C}$. El sobrenadante se neutralizó con Tris-base $(0,86 \mathrm{M})$. El paso adicional de crioprecipitación se llevó a cabo por almacenamiento de las muestras neutralizadas a $-20^{\circ} \mathrm{C}$ por una hora. Luego de este tiempo, por centrifugación a $3.000 \mathrm{rpm}, 30$ minutos a $4{ }^{\circ} \mathrm{C}$ se separaron las proteínas precipitadas. El sobrenadante finalmente obtenido, se analizó mediante RIA (dilución 1:28, suero, y 1:14, extractos de tejidos). La concentración de hormona se obtuvo mediante el programa RIA-Star 
(15). Para los extractos de tejidos, la concentración final es dada en $\mathrm{ng} / \mathrm{mg}$ de proteína total. El porcentaje de recuperación de IGF-I exógeno en los tejidos fue de $70 \%$ y para los sueros de $91 \%$.

\section{Determinación de proteína total}

Se realizó por el método de MicroKjeldahl en todos los tejidos analizados.

\section{Resultados y discusión \\ Contenido sérico de IGF-I}

El principal problema en el análisis del IGF-I en suero y fluidos biológicos es su asociación con las proteínas de unión (IGFBP). Un caso frecuente de interferencia ocurre cuando los anticuerpos están dirigidos contra epítopes parcialmente bloqueados por las IGFBP, ocasionando subvaloraciones del péptido. Las IGFBP también compiten con los anticuerpos por su unión al trazador (radioligando), ocasionando en este caso sobreestimaciones. Por tanto, para una determinación confiable se requiere disociar y separar el péptido de sus proteínas de unión antes del análisis. Se han desarrollado varios sistemas con este propósito, el más frecuente es la extracción ácidoetanólica (AE) que aunque corrientemente se emplea para muestras de suero humano, se ha observado que no elimina todas las IGFBP, en particular IGFBP-1 (11). La adición de una cromatografía de filtración en gel a $\mathrm{pH}$ ácido produce una mejor separación (12), pero resulta inconveniente cuando se analizan varias muestras además de que el tamaño de la misma es relativamente grande. En el caso de tejidos, la capacidad de remoción de las IGFBP por extracción ácido-etanol, no ha sido verificada.

En este trabajo se comparó el tratamiento AE y la inclusión de un paso de crioprecipitación $(A E C)$, en las determinaciones de IGF-I total en muestras de suero y algunos tejidos de rata. Se empleó como trazador en el RIA, la forma truncada Des(1-3) IGF-I, la cual posee una afinidad menor por las proteínas de unión, lo cual reduce aún más la interferencia de estas proteínas.

En el cuadro 1 se muestran los resultados del análisis de IGF-I en muestras sin tratamiento, así como con las extracciones $\mathrm{AE}$ y $\mathrm{AEC}$, en suero humano y de rata.

Cuadro 1. Concentración sérica de IGF-I en suero de rata normal y suero humano adulto normal. El contenido de IGF-I se determinó por RIA específico en muestras nativas (ST) y con los tratamientos de eliminación de IGFBP (AE y AEC). Los valores mostrados son el promedio \pm SEM de 10 determinaciones y están dadas en $\mathrm{ng} / \mathrm{mL}$.

\begin{tabular}{ccc}
\hline Tratam & Suero rata & Suero humano \\
\hline ST & $110,7 \pm 13,2$ & $94,3 \pm 13,2$ \\
AE & $410,6 \pm 30,2$ & $332,6 \pm 12,1$ \\
AEC & $511,2 \pm 34,6$ & $398,9 \pm 11,4$ \\
\hline
\end{tabular}

Como se observa, la extracción AEC permite cuantificar aproximadamente $20 \%$ más de IGF-I en comparación con la sola extracción $\mathrm{AE}$, indicando que este último tratamiento tiene una capacidad limitada de separación de las IGFBP en los dos tipos de suero. La crioprecipitación separa una fracción adicional de dichas proteínas, mejorando el análisis y permitiendo estimaciones del péptido más cercanas a su contenido real. Por otra parte, las concentraciones encontradas para las muestras sin tratamiento -en promedio, $24 \%$ del IGF-I totalrepresentan la fracción libre del péptido disponible para ser reconocido por las células blanco. Esta determinación tiene significado fisiológico, puesto que la regulación del equilibrio entre las formas libre y enlazada es esencial para el mantenimiento de la homeostasis celular. Esta regulación es muy compleja y sus mecanismos no han sido aún dilucidados. Existen diversas proteasas en suero que actúan a nivel de los complejos ternarios y binarios entre el IGF-I y sus IGFBP, que generan péptido libre ya sea por acción sobre las proteínas de unión $(16,17)$ o sobre el IGF-I. Otro mecanismo es a través de la formación de la variante Des(1-3) IGF-I por conversión enzimática del IGF-I intacto, el cual ha sido detectado en suero y tejidos de rata (18), como también en suero humano (19).

\section{Contenido tisular de IGF-I}

La cuantificación del IGF-I total en algunos tejidos de rata adulta joven se muestra en la figura 1. 


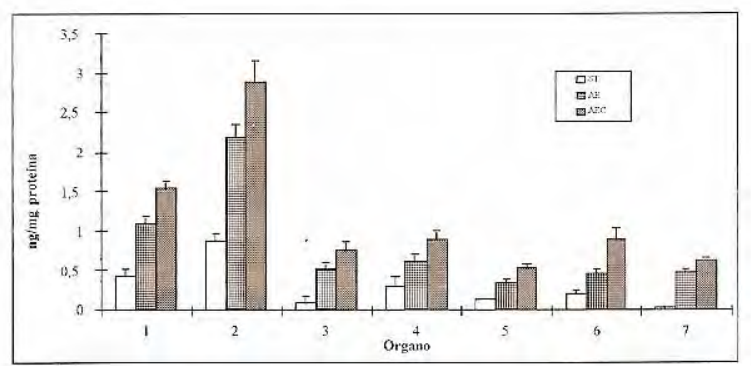

Figura 1. Concentración tisular de IGF-I en ratas normales. La técnica de RIA se utilizó para cuantificar IGFI en extractos de tejidos sin eliminación total de IGFBP (ST) y con la remoción de estas proteínas por los métodos $A E$ y AEC. Los valores mostrados corresponden al promedio de las determinaciones hechas en 5 animales (mínimo por triplicado) y son expresadas como ng IGF-I/mg de proteína total \pm SEM. Tejidos: 1) Hígado, 2) Riñón, 3) Corazón, 4) Pulmón, 5) Timo, 6) Bazo, y 7) Músculo esquelético.

Se observa que, en todos los tejidos analizados exceptuando el músculo esquelético, se detectan niveles variables de IGF-I libre (10-30\%) y concentraciones totales que son específicas de tejido. Al igual que en el caso de los análisis en suero, los tratamientos AE y AEC liberaron contenidos mayores del péptido, indicando que probablemente el IGF-I en dichos tejidos se encuentra mayormente enlazado a las proteínas de unión. Mediante ensayos de Western ligand blot y cromatografía de filtración en gel, se ha evidenciado que estos complejos son de bajo peso molecular y contienen principalmente IGFBP-1 e IGFBP-2 (publicación en preparación), lo cual está de acuerdo con el hecho de que la subunidad ácido-lábil necesaria para la formación de los complejos ternarios, solo se ha encontrado en suero (20).

En cuanto a la medición de los contenidos del IGF-I total en los tejidos de rata seleccionados, se encontraron niveles variables dependiendo del tejido analizado. La síntesis de IGF-I se ha demostrado en una variedad de órganos y células y aunque sus funciones se desconocen en su mayor parte, se acepta que puede estar actuando como hormona autocrina/paracrina, además de su acción endocrina estimulada por la hormona de crecimiento $(\mathrm{GH})(7,8)$. Los resultados obtenidos muestran que el riñón es el órgano con el mayor contenido de IGF-I total, segui- do por el hígado, mientras que los valores más bajos se encontraron en los órganos linfoides, timo y bazo. Aunque en hígado la concentración del mRNA de IGF-I es mucho más elevada que en los otros órganos, el péptido allí sintetizado no se almacena sino que pasa mayormente a la circulación. Llama la atención los altos niveles encontrados en riñón que no están de acuerdo con los contenidos de mRNA descritos para este órgano y que son entre 10 y 50 veces más bajos que los hepáticos. Resultados similares han sido encontrados por otros autores $(8,21$, 22) sugiriendo un transporte transcapilar del IGF-I circulante, mediado por las proteínas de unión. Además, el riñón es probablemente el sitio principal de degradación del IGF-I (23).

En órganos linfoides se encontraron las concentraciones más bajas del péptido; sin embargo, es posible que el mismo esté cumpliendo funciones a nivel de diferenciación y proliferación de linfocitos. Algunos estudios indican que linfocitos humanos periféricos pueden sintetizar IGF, así como sus receptores y cuatro diferentes IGFBP (24), lo que está de acuerdo con los resultados encontrados. Finalmente, está establecido que el músculo esquelético es un órgano blanco de la hormona de crecimiento la cual regula la síntesis local de IGF-I y donde, además, ocurre captación del péptido desde la circulación (22) lo cual está de acuerdo con los resultados aquí obtenidos que muestran un alto porcentaje de IGF-I en forma enlazada.

En conclusión, el método aquí descrito permite extraer y cuantificar el IGF-I en tejidos y suero de rata por medio de RIA. El paso adicional de crioprecipitación mejora la confiabilidad de las determinaciones, acercándose al contenido real del péptido. Mediante este método es posible iniciar estudios de regulación hormonal o nutricional del eje GH/IGF-I tomando como modelo experimental la rata.

\section{Agradecimientos}

Los autores expresan sus agradecimientos a la Universidad de Uppsala (Suecia), a Colciencias y al Departamento de Química de la Universidad Nacional. 


\section{Referencias}

1. Blundell TL, Bedarkar S, Rinderknecht $E$, et al. Insulin-like growth factor: a model of the tertiary structure accounting for immunoreactivity and receptor binding. Proc Natl Acad Sci USA 1978;75:180.

2. Yang $\mathrm{Y}$, Wang J, Orlowski $\mathrm{C}$, et al. Structure, specificity and regulation of the insulin-like growth factor-binding proteins in adult rat serum. Endocr 1989;125:1540.

3. Cohen P, Rosenfeld R. Physiological and clinical relevance of the insulin-like growth factor binding proteins. Curr Opin Pediat 1994;6:462.

4. Clemmons DR, Underwood LE. Nutritional regulation of IGF-I and IGF binding proteins. Annu Rev Nutr 1991;11:393.

5. Greenspan FS. Capítulo 7. En: Basic and clinical endocrinology. New Jersey: Prentice Hall International Inc.; 1991. p. 133.

6. Humbel RE. Review: insulin-like growth factors I and II. Eur J Biochem 1990;190:445.

7. Murphy L, Bell G, Friesen H. Tissue distribution of insulin-like growth factor-I and II messenger ribonucleic acid in adult rat. Endocr 1987;120:1279.

8. Lund $P$, Moats-Staats $B$, Hynes $M$, et al. Somatomedin-C/insulin-like growth factor-I and insulin-like growth factor-II mRNAs in rat fetal and adult tissues. J Biol Chem 1986;261:14539.

9. Goldstein S, Phillips L. Extraction and nutritional/ hormonal regulation of tissue insulin-like growth factor-I activity. J Biol Chem 1991;266:14725.

10. Breier BH, Gallager BW, Gluckman PD. Radioimmunoassay for insulin-like growth factor. I:Solution to some potential problems and pitfalls. J Endocr 1991;128:347.

11. Mesiano S, Young IR, Browne CA, et al. Failure of acid-ethanol treatment to prevent interference by binding proteins in radioligand assays for the insulinlike growth factors. J Endocr 1983;119:453.

12. Bang P, Erickson U, Sara V, Wivall I, Hall K. Comparison of acid ethanol extraction and acid gel filtration prior to IGF-I and IGF-II radioimmunoassay: improvement of determinations in acid ethanol extracts by the use of truncated IGF-I as radioligand. Acta Endocr (Copenh) 1991;124:620.

13. Kabi Pharmacia. Quantification of insulin-like growth factor-1 in serum by means of radioimmunoassay. Short description of IGF-I tissue extraction and RIA. Stockholm, Sweden; 1992.
14. Rodríguez SC, Sánchez-Gómez M, Anzola C; Lombana N, Pérez E. Método de radioinmunoanálisis para determinación del factor de crecimiento similar a la insulina-I (IGF-I) en suero humano. Información Tecnológica 1997;8(4):145.

15. Cekan SZ. Reliability of steroid radioimmunoassay (thesis). Uppsala: Universidad de Uppsala; 1976.

16. Jones JI, Clemmons DR. Insulin-like growth factors and their binding proteins: biological actions. Endocr Rev 1995;16:3.

17. Zapf Jürgen. Physiological role of the insulin-like growth factor binding proteins. Eur $\mathrm{J}$ Endocrinol 1995;132:645.

18. Yamamoto H, Murphy LJ. Enzymatic conversion of IGF-I to des(1-3)IGF-I in rat serum and tissues: a further potential site of growth hormone regulation of IGF-I action. J Endocr 1995;146:141.

19. Yamamoto H, Murphy LJ, Sohmiya M, et al. Serum N-terminal IGF-I protease activity in normal adults. $10^{\text {th }}$ International Congress of Endocrinology, San Francisco, 1996.

20. Ooi GT, cohen FJ, Tseng LY-H, et al. Growth hormone stimulates transcription of the gene for the acid-labile subunit of the $150 \mathrm{kDa}$ IGF-binding protein complex. $10^{\text {th }}$ International Congress of Endocrinology, San Francisco, 1996.

21. Martina A, Gosteli P, Kaspar $\mathbf{H}$, et al. Expression and regulation of insulin-like growth factor-I (IGF-I) and IGF-binding proteins messenger ribonucleic acid levels in tissues of hypophysectomized rats infused with IGF-1 and growth hormone. Endocr 1994;135: 2558.

22. Skottner A, Clark RG, Fryklund L, et al. Growth responses in a mutant dwarf rat to human growth hormone and recombinant human Insulin-like growth factor-I. En: Human growth hormone variants and insulin-like growth factor. I:Effects on growth parameters and peripheral nerve regeneration in rats (tesis). Stockholm, Sweden, 1988.

23. D'Ercole A, Stiles AD, Underwood LE. Tissues concentration of somatomedin $\mathrm{C}$ : further evidence for multiple sites of synthesis and paracrine or autocrine mechanics of actions. Proc Natl Acad Sci USA 1984; $81: 935$.

24. Nyman T, Pekonen F. The expression of the insulinlike growth factors and their binding proteins in normal human lymphocytes. Act Endocr 1993;128: 168. 